\title{
Existence and Uniqueness of Positive Solution for a Fractional Dirichlet Problem with Combined Nonlinear Effects in Bounded Domains
}

\author{
Imed Bachar' ${ }^{1}$ and Habib Mâagli ${ }^{2}$ \\ ${ }^{1}$ Mathematics Department, College of Sciences, King Saud University, \\ P.O. Box 2455, Riyadh 11451, Saudi Arabia \\ ${ }^{2}$ Mathematics Department, College of Sciences and Arts, King Abdulaziz University, Rabigh Campus, P.O. Box 344, \\ Rabigh 21911, Saudi Arabia \\ Correspondence should be addressed to Imed Bachar; abachar@ksu.edu.sa
}

Received 20 February 2013; Accepted 10 July 2013

Academic Editor: Daniel C. Biles

Copyright (C) 2013 I. Bachar and H. Mâagli. This is an open access article distributed under the Creative Commons Attribution License, which permits unrestricted use, distribution, and reproduction in any medium, provided the original work is properly cited.

We prove the existence and uniqueness of a positive continuous solution to the following singular semilinear fractional Dirichlet problem $(-\Delta)^{\alpha / 2} u=a_{1}(x) u^{\sigma_{1}}+a_{2}(x) u^{\sigma_{2}}$, in $D \lim _{x \rightarrow z \in \partial D}(\delta(x))^{1-(\alpha / 2)} u(x)=0$, where $0<\alpha<2, \sigma_{1}, \sigma_{2} \in(-1,1), D$ is a bounded $C^{1,1}$-domain in $\mathbb{R}^{n}, n \geq 2$, and $\delta(x)$ denotes the Euclidian distance from $x$ to the boundary of $D$. The nonnegative weight functions $a_{1}, a_{2}$ are required to satisfy certain hypotheses related to the Karamata class. We also investigate the global behavior of such solution.

\section{Introduction}

In the last two decades, several studies have been performed for the so-called fractional Laplacian, $(-\Delta)^{\alpha / 2}, 0<\alpha<2$, which can be defined by the integral representation

$$
(-\Delta)^{\alpha / 2} u(x)=c_{n, \alpha} \lim _{\varepsilon \searrow 0} \int_{(|x-y|>\varepsilon)} \frac{u(x)-u(y)}{|x-y|^{n+\alpha}} d y,
$$

where $c_{n, \alpha}=\left(\alpha 2^{\alpha-1} / \pi^{n / 2}\right)(\Gamma((n+\alpha) / 2) / \Gamma(1-(\alpha / 2)))$ is a normalization constant; see, for instance, $[1,2]$. From a probabilistic point of view, the fractional Laplacian appears as the infinitesimal generator of the stable Lévy process $[3,4]$; see also [5]. The fractional powers of the Laplacian arise in a numerous variety of equations in mathematical physics and related fields (see, for instance, [6-11] and the references therein). Motivation from mechanics appears in the Signorini problem (cf. $[12,13])$. And there are applications in fluid mechanics, (cf. [14]). The systematic study of the corresponding PDE models is more recent and many of the results have arisen in the last decade. The linear or quasilinear elliptic theory has been actively studied recently in the works of Caffarelli and collaborators [15, 16], Kassmann [17], Silvestre [18], and many others. The standard linear evolution equation involving fractional diffusion is

$$
\frac{\partial u}{\partial t}+(-\Delta)^{\alpha / 2} u=0
$$

This is a model of the so-called anomalous diffusion, a much studied topic in physics, probability, and finance (see [1923 ] and their references). For more applications, we refer the reader to the survey papers $[24,25]$.

Throughout this paper, we consider a bounded $C^{1,1}$ domain $D$ in $\mathbb{R}^{n}, n \geq 2$, and we denote by $\delta(x)$ the Euclidian distance from $x$ to the boundary of $D$. For two nonnegative functions $f$ and $g$ defined on a set $S$, the notation $f(x) \approx$ $g(x), x \in S$, means that there exists $c>0$ such that $(1 / c) f(x) \leq g(x) \leq c f(x)$, for all $x \in S$. 
Recently, in [26], the authors considered the following problem:

$$
\begin{cases}(-\Delta)^{\alpha / 2} u=\varphi(\cdot, u) & \text { in } D \text { (in the sense of distributions), } \\ u>0 & \text { in } D, \\ \lim _{x \rightarrow z \in \partial D}(\delta(x))^{1-(\alpha / 2)} u(x)=0, & \end{cases}
$$

where $0<\alpha<2$ and $\varphi$ is a positive measurable function in $D \times(0, \infty)$ satisfying the following:

$\left(\mathrm{A}_{1}\right)$ the map $t \rightarrow \varphi(x, t)$ is continuous and nonincreasing in $(0, \infty)$, for $x \in D$;

$\left(\mathrm{A}_{2}\right)$ for each $c>0$, the function $x \rightarrow$ $(\delta(x))^{1-(\alpha / 2)} \varphi\left(x, c(\delta(x))^{(\alpha / 2)-1}\right)$ is in $K^{\alpha}(D)$ (see Definition 1 below).

They have proved that problem (3) has a positive continuous solution $u$ in $D$ satisfying, for each $x \in D$,

$$
u(x)=\int_{D} G_{D}^{\alpha}(x, y) \varphi(y, u(y)) d y,
$$

where $G_{D}^{\alpha}(x, y)$ denotes the Green function of the fractional Laplacian $(-\Delta)^{\alpha / 2}$ in $D$. However they have not investigated the asymptotic behavior of such solution.

As a typical example of function $\varphi$ satisfying $\left(\mathrm{A}_{1}\right)$ and $\left(\mathrm{A}_{2}\right)$, we quote $\varphi(x, u)=a(x) u^{\sigma}$, where $\sigma \leq 0$ and $a$ is a positive measurable function in $D$ such that the function

$$
x \longrightarrow(\delta(x))^{((\alpha / 2)-1)(\sigma-1)} a(x)
$$

belongs to the Kato class $K^{\alpha}(D)$ defined as follows.

Definition 1 (see [26]). A Borel measurable function $q$ in $D$ belongs to the Kato class $K^{\alpha}(D)$ if

$$
\lim _{r \rightarrow 0}\left(\sup _{x \in D} \int_{(|x-y| \leq r) \cap D}\left(\frac{\delta(y)}{\delta(x)}\right)^{\alpha / 2} G_{D}^{\alpha}(x, y)|q(y)| d y\right)=0 .
$$

It has been proved in [26] that the function

$$
x \longrightarrow(\delta(x))^{-\lambda} \text { belongs to } K^{\alpha}(D) \quad \text { iff } \lambda<\alpha .
$$

For more examples of functions belonging to $K^{\alpha}(D)$, we refer to [26]. Note that for the classical case (i.e., $\alpha=2$ ) the class $K^{2}(D)$ was introduced and studied in [27].

On the other hand, Chemmam et al. considered in [28] the following semilinear fractional Dirichlet problem:

$$
\begin{cases}(-\Delta)^{\alpha / 2} u=a(x) u^{\sigma} & \text { in } D(\text { in the sense of distributions), } \\ u>0 & \text { in } D, \\ \lim _{x \rightarrow z \in \partial D}(\delta(x))^{1-(\alpha / 2)} u(x)=0, & \end{cases}
$$

where $0<\alpha<2, \sigma<1$, and $a$ satisfies the following hypothesis:

$$
\begin{array}{r}
\left(\mathrm{H}_{0}\right) a \in C_{\mathrm{loc}}^{\gamma}(D), 0<\gamma<1, \text { satisfying } D, \\
a(x) \approx(\delta(x))^{-\lambda} L(\delta(x)),
\end{array}
$$

where $\lambda<(\alpha / 2)(1+\sigma)+1-\sigma$ and $L$ belongs to the Karamata class $\mathscr{K}$ defined as follows.
Definition 2. The class $\mathscr{K}$ is the set of all the Karamata functions $L$ defined on $(0, \eta]$ by

$$
L(t):=c \exp \left(\int_{t}^{\eta} \frac{z(s)}{s} d s\right)
$$

where $\eta>\operatorname{diam}(D), c>0$, and $z \in C([0, \eta])$ such that $z(0)=$ 0 .

As a typical example of a function belonging to the class $\mathscr{K}$ (see [29-31]), we quote

$$
L(t)=\prod_{k=1}^{m}\left(\log _{k}\left(\frac{\omega}{t}\right)\right)^{-\xi_{k}}
$$

where $\xi_{k}$ are real numbers, $\log _{k} x=\log \circ \log \circ \cdots \log x$ ( $k$ times), and $\omega$ is a sufficiently large positive real number such that $L$ is defined and positive on $(0, \eta]$.

Using a fixed-point argument, the authors have proved in [28] the existence and uniqueness of a positive continuous solution $u$ for (8) satisfying, for $x \in D$,

$$
u(x) \approx(\delta(x))^{\min (\alpha / 2,(\alpha-\lambda) /(1-\sigma))} \Psi_{L, \lambda, \sigma}(\delta(x)),
$$

where the function $\Psi_{L, \lambda, \sigma}$ is defined on $(0, \eta)$ by

$$
\Psi_{L, \lambda, \sigma}(t):= \begin{cases}(L(t))^{1 /(1-\sigma)}, & \frac{\alpha}{2}(1+\sigma)<\lambda \\ \left(\int_{t}^{\eta} \frac{L(s)}{s} d s\right)^{1 /(1-\sigma)}, & \text { if } \lambda=\frac{\alpha}{2}(1+\sigma), 1-\sigma, \\ 1, & \text { if } \lambda<\frac{\alpha}{2}(1+\sigma) .\end{cases}
$$

In particular, they have extended the results of $[32,33]$.

In the present paper, we aim at studying the following fractional nonlinear problem involving both singular and sublinear nonlinearities with the reformulated Dirichlet boundary condition:

$$
\begin{cases}(-\Delta)^{\alpha / 2} u=a_{1}(x) u^{\sigma_{1}}+a_{2}(x) u^{\sigma_{2}} & \text { in } D \text { (in the sense of } \\ \text { distributions) }\end{cases}
$$

where $0<\alpha<2$ and $\sigma_{1}, \sigma_{2} \in(-1,1)$. We will address the question of existence, uniqueness, and global behavior of a positive continuous solution to problem (14). 
In the elliptic case (i.e., $\alpha=2$ ), problems related to (14) have been studied by several authors (see, e.g., [34-39] and references therein). Using the subsupersolution method, the authors in [36] have established the existence and uniqueness of a positive continuous solution to (14) for $\alpha=2, \sigma_{1}, \sigma_{2}<$ 1 , where the functions $a_{1}, a_{2}$ are required to satisfy some adequate assumptions related to the Karamata class $\mathscr{K}$.

Here, our goal is to study problem (14) for $0<\alpha<2$. To this end, we assume that the potential functions $a_{1}, a_{2}$ satisfy the following hypothesis.

(H) for $i \in\{1,2\}, a_{i} \in C_{\mathrm{loc}}^{\gamma}(D), 0<\gamma<1$, and satisfies, for $x \in D$,

$$
a_{i}(x) \approx(\delta(x))^{-\lambda_{i}} L_{i}(\delta(x)),
$$

where $\lambda_{i}<(\alpha / 2)\left(1+\sigma_{i}\right)+1-\sigma_{i}$ and $L_{i} \in \mathscr{K}$ defined on $(0, \eta]$ with $\eta>\operatorname{diam}(D)$.

As it turns out, estimates (12) depend closely on $\min (\alpha / 2,(\alpha-\lambda) /(1-\sigma))$. Also, as it will be seen, the numbers

$$
\beta_{1}:=\min \left(\frac{\alpha}{2}, \frac{\alpha-\lambda_{1}}{1-\sigma_{1}}\right), \quad \beta_{2}:=\min \left(\frac{\alpha}{2}, \frac{\alpha-\lambda_{2}}{1-\sigma_{2}}\right)
$$

play an important role in the combined effect of singular and superlinear nonlinearities in (14) and lead to a competition. It is not obvious which wins, essentially in the estimates of solution. From here on and without loss of generality, we may assume that $\left(\alpha-\lambda_{1}\right) /\left(1-\sigma_{1}\right) \leq\left(\alpha-\lambda_{2}\right) /\left(1-\sigma_{2}\right)$ and we introduce the function $\theta$ defined on $(0, \eta)$ by

$$
\theta(t)= \begin{cases}t^{\beta_{1}} \Psi_{L_{1}, \lambda_{1}, \sigma_{1}}(t) & \text { if } \beta_{1}<\beta_{2} \\ t^{\beta_{1}}\left(\Psi_{L_{1}, \lambda_{1}, \sigma_{1}}(t)+\Psi_{L_{2}, \lambda_{2}, \sigma_{2}}(t)\right) & \text { if } \beta_{1}=\beta_{2} .\end{cases}
$$

For an explicit form of the function $\theta$, see (36). by

Throughout this paper, we define the potential kernel $G_{D}^{\alpha}$

$G_{D}^{\alpha} f(x):=\int_{D} G_{D}^{\alpha}(x, y) f(y) d y, \quad$ for $x \in D, f \in B^{+}(D)$,

where $B^{+}(D)$ denotes the set of the nonnegative Borel measurable functions in $D$.

Our main results are the following.

Theorem 3. Let $\sigma_{1}, \sigma_{2} \in(-1,1)$ and assume $(H)$. Then one has, for $x \in D$,

$$
G_{D}^{\alpha}\left[a_{1} \theta^{\sigma_{1}}(\delta(\cdot))+a_{2} \theta^{\sigma_{2}}(\delta(\cdot))\right](x) \approx \theta(\delta(x)) .
$$

Using Theorem 3 and the Schauder fixed-point theorem, we will prove the following.
Theorem 4. Let $\sigma_{1}, \sigma_{2} \in(-1,1)$ and assume $(H)$. Then problem (14) has a unique positive continuous solution $u$ in $D$ satisfying, for $x \in D$,

$$
u(x) \approx \theta(\delta(x)) .
$$

In particular, we generalize the result obtained in [36] to the fractional setting and we recover the result obtained in [28].

The content of this paper is organized as follows. In Section 2, we collect some properties of functions belonging to the Karamata class $\mathscr{K}$ and the Kato class $K^{\alpha}(D)$, which are useful to establish our results. In Section 3, we prove our main results.

As usual, we denote by $C_{0}(D)$ the set of continuous functions in $\bar{D}$ vanishing continuously on $\partial D$. Note that $C_{0}(D)$ is a Banach space with respect to the uniform norm $\|u\|_{\infty}=\sup _{x \in D}|u(x)|$. As in the elliptic case, if $f \in B^{+}(D)$ satisfies $\int_{D}(\delta(y))^{\alpha / 2} f(y) d y<\infty$, then the functions $f$ and $G_{D}^{\alpha} f$ are in $L_{\text {loc }}^{1}(D)$ and we have in the distributional sense

$$
(-\Delta)^{\alpha / 2} G_{D}^{\alpha} f=f, \quad \text { in } D .
$$

\section{The Karamata Class $\mathscr{K}$ and the Kato Class $K^{\alpha}(D)$}

We collect in this paragraph some properties of the Karamata class $\mathscr{K}$ and the Kato class $K^{\alpha}(D)$. We recall that a function $L$ defined on $(0, \eta]$ belongs to the class $\mathscr{K}$ if

$$
L(t):=c \exp \left(\int_{t}^{\eta} \frac{z(s)}{s} d s\right)
$$

where $\eta>\operatorname{diam}(D), c>0$, and $z \in C([0, \eta])$ such that $z(0)=$ 0 .

Proposition 5 (see $[30,31])$. (i) A function $L$ is in $\mathscr{K}$ if and only if $L$ is a positive function in $C^{1}((0, \eta])$ such that

$$
\lim _{t \rightarrow 0^{+}} \frac{t L^{\prime}(t)}{L(t)}=0 .
$$

(ii) Let $L_{1}, L_{2} \in \mathscr{K}, p \in \mathbb{R}$. Then one has

$$
L_{1}+L_{2} \in \mathscr{K}, \quad L_{1} L_{2} \in \mathscr{K}, \quad L_{1}^{p} \in \mathscr{K} .
$$

(iii) Let $L \in \mathscr{K}$ and $\varepsilon>0$. Then one has

$$
\lim _{t \rightarrow 0^{+}} t^{\varepsilon} L(t)=0 .
$$

Applying Karamata's theorem (see [30, 31]), we get the following.

Lemma 6. Let $\mu \in \mathbb{R}$ and let $L$ be a function in $\mathscr{K}$. One has the following:

(i) if $\mu<-1$, then $\int_{0}^{\eta} s^{\mu} L(s) d s$ diverges and $\int_{t}^{\eta} s^{\mu} L(s)$ $d s \sim_{t \rightarrow 0^{+}}\left(-t^{1+\mu} L(t)\right) /(\mu+1)$; 
(ii) if $\mu>-1$, then $\int_{0}^{\eta} s^{\mu} L(s) d s$ converges and $\int_{0}^{t} s^{\mu} L(s)$ $d s \sim_{t \rightarrow 0^{+}}\left(t^{1+\mu} L(t)\right) /(\mu+1)$.

Lemma 7 (see [36]). Let $L$ be a function in $\mathscr{K}$. Then one has

$$
\lim _{t \rightarrow 0^{+}} \frac{L(t)}{\int_{t}^{\eta}(L(s) / s) d s}=0 .
$$

In particular

$$
t \rightarrow \int_{t}^{\eta} \frac{L(s)}{s} d s \in \mathscr{K}
$$

Proposition 8 (see $[40,41])$. For $(x, y) \in D \times D$, one has

$$
G_{D}^{\alpha}(x, y) \approx|x-y|^{\alpha-n} \min \left(1, \frac{(\delta(x) \delta(y))^{\alpha / 2}}{|x-y|^{\alpha}}\right) .
$$

Proposition 9 (see [26, Corollary 6]). Let q be a nonnegative function in $K^{\alpha}(D)$; then the family of functions

$$
\Lambda_{q}=\left\{x \longrightarrow \int_{D}\left(\frac{\delta(y)}{\delta(x)}\right)^{(\alpha / 2)-1} G_{D}^{\alpha}(x, y) f(y) d y,|f| \leq q\right\}
$$

is uniformly bounded and equicontinuous in $\bar{D}$. Consequently $\Lambda_{q}$ is relatively compact in $C_{0}(D)$.

\section{Proofs of the Main Results}

In this section we aim at proving Theorems 3 and 4 . To this end, we need the following lemmas.

\subsection{Technical Lemmas}

Lemma 10. For $r, s>0$, one has

$$
\begin{aligned}
& 2^{-\max \left(1-\sigma_{1}, 1-\sigma_{2}\right)}(r+s) \\
& \quad \leq r^{1-\sigma_{1}}(r+s)^{\sigma_{1}}+s^{1-\sigma_{2}}(r+s)^{\sigma_{2}} \leq 2(r+s) .
\end{aligned}
$$

Proof. Let $r, s>0$ and put $t=r /(r+s)$. Since $0 \leq t \leq 1$, then we get obviously

$$
2^{-\max \left(1-\sigma_{1}, 1-\sigma_{2}\right)} \leq t^{1-\sigma_{1}}+(1-t)^{1-\sigma_{2}} \leq 2 .
$$

Lemma 11 provides sharp estimates on some Riesz potential functions.

Lemma 11 (see [28, Proposition 3.1]). Let $\mu \leq(\alpha / 2)+1$ and let $L$ be a function in $\mathscr{K}$ such that $\int_{0}^{\eta} t^{(\alpha / 2)-\mu} L(t) d t<\infty$. Let $q$ be a positive measurable function in $D$ such that, for $x \in D$,

$$
q(x) \approx(\delta(x))^{-\mu} L(\delta(x)) .
$$

Then, for $x \in D$, one has

$$
G_{D}^{\alpha} q(x) \approx \psi(\delta(x))
$$

where $\psi$ is the function defined on $(0, \eta)$ by

$$
\psi(t):= \begin{cases}t^{(\alpha / 2)-1} \int_{0}^{t} \frac{L(s)}{s} d s, & \text { if } \mu=\frac{\alpha}{2}+1, \\ t^{\alpha-\mu} L(t), & \text { if } \frac{\alpha}{2}<\mu<\frac{\alpha}{2}+1, \\ t^{\alpha / 2} \int_{t}^{\eta} \frac{L(s)}{s} d s, & \text { if } \mu=\frac{\alpha}{2}, \\ t^{\alpha / 2}, & \text { if } \mu<\frac{\alpha}{2} .\end{cases}
$$

Lemma 12. Assume $(H)$. Let $u$ be a continuous function in $D$ such that, for $x \in D, u(x) \approx \theta(\delta(x))$. Then $u$ is a solution of problem (14) if and only if

$$
u(x)=\int_{D} G_{D}^{\alpha}(x, y)\left[a_{1}(y) u^{\sigma_{1}}(y)+a_{2}(y) u^{\sigma_{2}}(y)\right] d y,
$$

$x \in D$.

Proof. Assume $(\mathrm{H})$. First we will give an explicit form of the function $\theta$. We recall that, for $i \in\{1,2\}, \lambda_{i}<(\alpha / 2)\left(1+\sigma_{i}\right)+$ $1-\sigma_{i}$ and $\beta_{i}:=\min \left(\alpha / 2,\left(\alpha-\lambda_{i}\right) /\left(1-\sigma_{i}\right)\right)$. Since $\beta_{1}<\beta_{2}$ is equivalent to $\left(\alpha-\lambda_{1}\right) /\left(1-\sigma_{1}\right)<\left(\alpha-\lambda_{2}\right) /\left(1-\sigma_{2}\right)$ and $(\alpha / 2)\left(1+\sigma_{1}\right)<\lambda_{1}$, we deduce that, for $t \in(0, \eta)$, we have

$$
\theta(t)=\left\{\begin{array}{lr}
t^{\left(\alpha-\lambda_{1}\right) /\left(1-\sigma_{1}\right)}\left(L_{1}(t)\right)^{1 /\left(1-\sigma_{1}\right)}, & \text { if } \frac{\alpha-\lambda_{1}}{1-\sigma_{1}}<\frac{\alpha-\lambda_{2}}{1-\sigma_{2}}, \\
\frac{\alpha}{2}\left(1+\sigma_{1}\right)<\lambda_{1}, \\
t^{\left(\alpha-\lambda_{1}\right) /\left(1-\sigma_{1}\right)} L(t), & \text { if } \frac{\alpha-\lambda_{1}}{1-\sigma_{1}}=\frac{\alpha-\lambda_{2}}{1-\sigma_{2}}, \\
\frac{\alpha}{2}\left(1+\sigma_{1}\right)<\lambda_{1}, \\
t^{\alpha / 2} M(t), & \text { if } \lambda_{1}=\frac{\alpha}{2}\left(1+\sigma_{1}\right), \\
\left.t_{t} \frac{\lambda_{2}}{s} d s\right)^{1 /\left(1-\sigma_{1}\right)}, & \text { if } \lambda_{1}=\frac{\alpha}{2}\left(1+\sigma_{2}\right), \\
t^{\alpha / 2}\left(\int_{1}^{\eta}(s),\right. & \lambda_{2}<\frac{\alpha}{2}\left(1+\sigma_{2}\right), \\
t^{\alpha / 2} & \text { if } \lambda_{1}<\frac{\alpha}{2}\left(1+\sigma_{1}\right),
\end{array}\right.
$$

where

$$
\begin{gathered}
L(t):=\left(L_{1}(t)\right)^{1 /\left(1-\sigma_{1}\right)}+\left(L_{2}(t)\right)^{1 /\left(1-\sigma_{2}\right)} \\
M(t):=\left(\int_{t}^{\eta} \frac{L_{1}(s)}{s} d s\right)^{1 /\left(1-\sigma_{1}\right)}+\left(\int_{t}^{\eta} \frac{L_{2}(s)}{s} d s\right)^{1 /\left(1-\sigma_{2}\right)} .
\end{gathered}
$$

Now using the fact that $u(x) \approx \theta(\delta(x))$, we deduce by simple computation from hypothesis $(\mathrm{H}),(36)$, and Proposition 5 that

$$
a_{1}(x) u^{\sigma_{1}}(x)+a_{2}(x) u^{\sigma_{2}}(x) \approx(\delta(x))^{(\alpha / 2)-1} h(\delta(x)),
$$

where $h$ is defined in $(0, \eta)$ by 


$$
h(t):= \begin{cases}t^{1-(\alpha / 2)-\left(\left(\lambda_{1}-\alpha \sigma_{1}\right) /\left(1-\sigma_{1}\right)\right)}\left(L_{1}(t)\right)^{1 /\left(1-\sigma_{1}\right)}, & \text { if } \frac{\alpha-\lambda_{1}}{1-\sigma_{1}}<\frac{\alpha-\lambda_{2}}{1-\sigma_{2}}, \frac{\alpha}{2}\left(1+\sigma_{1}\right)<\lambda_{1}, \\ t^{1-(\alpha / 2)-\left(\left(\lambda_{1}-\alpha \sigma_{1}\right) /\left(1-\sigma_{1}\right)\right)}\left(L_{1} L^{\sigma_{1}}+L_{2} L^{\sigma_{2}}\right)(t), & \text { if } \frac{\alpha-\lambda_{1}}{1-\sigma_{1}}=\frac{\alpha-\lambda_{2}}{1-\sigma_{2}}, \frac{\alpha}{2}\left(1+\sigma_{1}\right)<\lambda_{1}, \\ t^{1-\alpha}\left(L_{1} M^{\sigma_{1}}+L_{2} M^{\sigma_{2}}\right)(t), & \text { if } \lambda_{1}=\frac{\alpha}{2}\left(1+\sigma_{1}\right), \lambda_{2}=\frac{\alpha}{2}\left(1+\sigma_{2}\right), \\ t^{1-\alpha} L_{1}(t)\left(\int_{t}^{\eta} \frac{L_{1}(s)}{s} d s\right)^{\sigma_{1} /\left(1-\sigma_{1}\right)}, & \text { if } \lambda_{1}=\frac{\alpha}{2}\left(1+\sigma_{1}\right), \lambda_{2}<\frac{\alpha}{2}\left(1+\sigma_{2}\right), \\ t^{1+(\alpha / 2)\left(\sigma_{1}-1\right)-\lambda_{1}} L_{1}(t)+t^{1+(\alpha / 2)\left(\sigma_{2}-1\right)-\lambda_{2}} L_{2}(t), & \text { if } \lambda_{1}<\frac{\alpha}{2}\left(1+\sigma_{1}\right) .\end{cases}
$$

We point out that for each case, the function $h(t)$ can be written as a sum of terms of the form $t^{-\mu} \widetilde{L}(t)$, where $\mu<\alpha$. By Proposition 5 and Lemma 7, we have $\widetilde{L} \in \mathscr{K}$. On the other hand, since by Proposition 5, the function $x \rightarrow$ $(\delta(x))^{(\alpha-\mu) / 2} \widetilde{L}(\delta(x))$ is positive and belongs to $C_{0}(D)$, then there exists $c>0$ such that for each $x \in D$

$$
0<(\delta(x))^{-\mu} \widetilde{L}(\delta(x)) \leq c(\delta(x))^{-(\alpha+\mu) / 2} .
$$

Hence we deduce from (7) that the function $x \rightarrow h(\delta(x))$ is in $K^{\alpha}(D)$.

Now using Proposition 9, we obtain that $x \rightarrow$ $(\delta(x))^{1-(\alpha / 2)} G_{D}^{\alpha}\left[a_{1} u^{\sigma_{1}}+a_{2} u^{\sigma_{2}}\right](x)$ is in $C_{0}(D)$. In particular, we have

$$
\begin{gathered}
\lim _{x \rightarrow z \in \partial D}(\delta(x))^{1-(\alpha / 2)} G_{D}^{\alpha}\left(a_{1} u^{\sigma_{1}}+a_{2} u^{\sigma_{2}}\right)(x)=0, \\
(-\Delta)^{\alpha / 2} G_{D}^{\alpha}\left(a_{1} u^{\sigma_{1}}+a_{2} u^{\sigma_{2}}\right)=a_{1}(x) u^{\sigma_{1}}+a_{2}(x) u^{\sigma_{2}}
\end{gathered}
$$

in $D$ (in the sense of distributions).

Consequently, it follows by (41) that $u$ is a weak continuous solution of problem (14) if and only if $u$ satisfies

$$
\begin{cases}(-\Delta)^{\alpha / 2}\left(u-G_{D}^{\alpha}\left(a_{1} u^{\sigma_{1}}+a_{2} u^{\sigma_{2}}\right)\right)=0 & \text { in } D \\ \lim _{x \rightarrow z \in \partial D}(\delta(x))^{1-(\alpha / 2)}\left(u-G_{D}^{\alpha}\left(a_{1} u^{\sigma_{1}}+a_{2} u^{\sigma_{2}}\right)\right)=0 . & \end{cases}
$$

We deduce by [26, Theorem 6] that $u-G_{D}^{\alpha}\left(a_{1} u^{\sigma_{1}}+a_{2} u^{\sigma_{2}}\right)=0$ in $D$. The proof is complete.

Lemma 13. For $i \in\{1,2\}$, let $L_{i} \in \mathscr{K}$ defined on $(0, \eta]$ with $\eta>\operatorname{diam}(D)$ and let $M$ be the function given by (37). Then one has, for $t \in(0, \eta)$,

$$
\int_{t}^{\eta} \frac{\left(L_{1} M^{\sigma_{1}}+L_{2} M^{\sigma_{2}}\right)(s)}{s} d s \approx M(t) .
$$

Proof. The proof can be found in [36].

Now we are ready to prove our main results.
3.2. Proof of Theorem 3. Assume (H). For $i \in\{1,2\}$, let $L_{i} \in \mathscr{K}$ defined on $(0, \eta]$ with $\eta>\operatorname{diam}(D)$ and define the nonnegative functions $b_{i}$ in $(0, \eta)$ by

$$
b_{i}(t)=\left(\int_{t}^{\eta} \frac{L_{i}(s)}{s} d s\right)^{1 /\left(1-\sigma_{i}\right)} .
$$

Let $\theta$ be the function given by (36). To prove Theorem 3, we distinguish the following cases.

Case 1. $\left(\alpha-\lambda_{1}\right) /\left(1-\sigma_{1}\right)<\left(\alpha-\lambda_{2}\right) /\left(1-\sigma_{2}\right)$ and $(\alpha / 2)\left(1+\sigma_{1}\right)<$ $\lambda_{1}<(\alpha / 2)\left(1+\sigma_{1}\right)+1-\sigma_{1}$.

Since $\theta(t)=t^{\left(\alpha-\lambda_{1}\right) /\left(1-\sigma_{1}\right)}\left(L_{1}(t)\right)^{1 /\left(1-\sigma_{1}\right)}$, then we have

$$
\begin{gathered}
a_{1}(x) \theta^{\sigma_{1}}(\delta(x)) \approx(\delta(x))^{\left(\alpha \sigma_{1}-\lambda_{1}\right) /\left(1-\sigma_{1}\right)}\left(L_{1}(\delta(x))\right)^{1 /\left(1-\sigma_{1}\right)}, \\
a_{2}(x) \theta^{\sigma_{2}}(\delta(x)) \\
\approx(\delta(x))^{\left(\left(\alpha-\lambda_{1}\right) /\left(1-\sigma_{1}\right)\right) \sigma_{2}-\lambda_{2}}\left(L_{2} L_{1}^{\sigma_{2} /\left(1-\sigma_{1}\right)}\right)(\delta(x)) .
\end{gathered}
$$

Using the fact that $\left(\alpha \sigma_{1}-\lambda_{1}\right) /\left(1-\sigma_{1}\right)<\left(\left(\alpha-\lambda_{1}\right) /\left(1-\sigma_{1}\right)\right) \sigma_{2}-$ $\lambda_{2}$, we deduce by Proposition 5 that

$$
\begin{aligned}
a_{1}(x) & \theta^{\sigma_{1}}(\delta(x))+a_{2}(x) \theta^{\sigma_{2}}(\delta(x)) \\
& \approx(\delta(x))^{\left(\alpha \sigma_{1}-\lambda_{1}\right) /\left(1-\sigma_{1}\right)}\left(L_{1}(\delta(x))\right)^{1 /\left(1-\sigma_{1}\right)} .
\end{aligned}
$$

Since, for $\mu=\left(\lambda_{1}-\alpha \sigma_{1}\right) /\left(1-\sigma_{1}\right) \in(\alpha / 2,(\alpha / 2)+1)$, we have $\int_{0}^{\eta} t^{(\alpha / 2)-\mu}\left(L_{1}(t)\right)^{1 /\left(1-\sigma_{1}\right)} d t<\infty$, then applying Lemma 11, we deduce that

$$
\begin{aligned}
G_{D}^{\alpha} & {\left[a_{1} \theta^{\sigma_{1}}(\delta(\cdot))+a_{2} \theta^{\sigma_{2}}(\delta(\cdot))\right](x) } \\
& \approx G_{D}^{\alpha}\left[(\delta(\cdot))^{\left(\alpha \sigma_{1}-\lambda_{1}\right) /\left(1-\sigma_{1}\right)}\left(L_{1}(\delta(\cdot))\right)^{1 /\left(1-\sigma_{1}\right)}\right](x) \\
& \approx(\delta(x))^{\left(\alpha-\lambda_{1}\right) /\left(1-\sigma_{1}\right)}\left(L_{1}(\delta(x))\right)^{1 /\left(1-\sigma_{1}\right)}=\theta(\delta(x)) .
\end{aligned}
$$

Case 2. $\left(\alpha-\lambda_{1}\right) /\left(1-\sigma_{1}\right)=\left(\alpha-\lambda_{2}\right) /\left(1-\sigma_{2}\right)$ and $(\alpha / 2)\left(1+\sigma_{1}\right)<$ $\lambda_{1}<(\alpha / 2)\left(1+\sigma_{1}\right)+1-\sigma_{1}$.

In this case $\theta(t)=t^{\left(\alpha-\lambda_{1}\right) /\left(1-\sigma_{1}\right)} L(t)$. Therefore

$$
a_{1}(x) \theta^{\sigma_{1}}(\delta(x)) \approx(\delta(x))^{\left(\alpha \sigma_{1}-\lambda_{1}\right) /\left(1-\sigma_{1}\right)}\left(L_{1} L^{\sigma_{1}}\right)(\delta(x)) .
$$


So we obtain by Proposition 5 and Lemma 11 with $\mu=\left(\lambda_{1}-\right.$ $\left.\alpha \sigma_{1}\right) /\left(1-\sigma_{1}\right) \in(\alpha / 2,(\alpha / 2)+1)$,

$$
G_{D}^{\alpha}\left[a_{1} \theta^{\sigma_{1}}(\delta(\cdot))\right](x) \approx(\delta(x))^{\left(\alpha-\lambda_{1}\right) /\left(1-\sigma_{1}\right)}\left(L_{1} L^{\sigma_{1}}\right)(\delta(x)) .
$$

Similarly, since $(\alpha / 2)\left(1+\sigma_{2}\right)<\lambda_{2}<(\alpha / 2)\left(1+\sigma_{2}\right)+1-\sigma_{2}$, we obtain

$$
\begin{aligned}
G_{D}^{\alpha}\left[a_{2} \theta^{\sigma_{2}}(\delta(\cdot))\right](x) \\
\quad \approx(\delta(x))^{\left(\alpha-\lambda_{2}\right) /\left(1-\sigma_{2}\right)}\left(L_{2} L^{\sigma_{2}}\right)(\delta(x)) \\
\quad \approx(\delta(x))^{\left(\alpha-\lambda_{1}\right) /\left(1-\sigma_{1}\right)}\left(L_{2} L^{\sigma_{2}}\right)(\delta(x)) .
\end{aligned}
$$

Hence by using (30), we deduce that

$$
\begin{aligned}
G_{D}^{\alpha} & {\left[a_{1} \theta^{\sigma_{1}}(\delta(\cdot))+a_{2} \theta^{\sigma_{2}}(\delta(\cdot))\right](x) } \\
& \approx(\delta(x))^{\left(\alpha-\lambda_{1}\right) /\left(1-\sigma_{1}\right)}\left(L_{1} L^{\sigma_{1}}+L_{2} L^{\sigma_{2}}\right)(\delta(x)) \\
& \approx(\delta(x))^{\left(\alpha-\lambda_{1}\right) /\left(1-\sigma_{1}\right)} L(\delta(x))=\theta(\delta(x)) .
\end{aligned}
$$

Case 3. If $\lambda_{1}=(\alpha / 2)\left(1+\sigma_{1}\right)$ and $\lambda_{2}=(\alpha / 2)\left(1+\sigma_{2}\right)$ and since $\theta(t)=t^{\alpha / 2} M(t)$, then we have

$$
\begin{aligned}
a_{1}(x) & \theta^{\sigma_{1}}(\delta(x))+a_{2}(x) \theta^{\sigma_{2}}(\delta(x)) \\
& \approx(\delta(x))^{-\alpha / 2}\left(L_{1} M^{\sigma_{1}}+L_{2} M^{\sigma_{2}}\right)(\delta(x)) .
\end{aligned}
$$

So by Proposition 5, Lemma 11 with $\mu=\alpha / 2$, and Lemma 13, we deduce that

$$
\begin{aligned}
G_{D}^{\alpha} & {\left[a_{1} \theta^{\sigma_{1}}(\delta(\cdot))+a_{2} \theta^{\sigma_{2}}(\delta(\cdot))\right](x) } \\
& \approx(\delta(x))^{\alpha / 2} \int_{\delta(x)}^{\eta} \frac{\left(L_{1} M^{\sigma_{1}}+L_{2} M^{\sigma_{2}}\right)(s)}{s} d s \\
& \approx(\delta(x))^{\alpha / 2} M(\delta(x))=\theta(\delta(x)) .
\end{aligned}
$$

Case 4. $\lambda_{1}=(\alpha / 2)\left(1+\sigma_{1}\right)$ and $\lambda_{2}<(\alpha / 2)\left(1+\sigma_{2}\right)$.

In this case $\theta(t)=t^{\alpha / 2} b_{1}(t)$. Since $\lambda_{2}-\left(\alpha \sigma_{2} / 2\right)<(\alpha / 2)$, we deduce by Proposition 5 that

$$
\begin{aligned}
a_{1}(x) & \theta^{\sigma_{1}}(\delta(x))+a_{2}(x) \theta^{\sigma_{2}}(\delta(x)) \\
\approx & (\delta(x))^{-\alpha / 2}\left(L_{1} b_{1}^{\sigma_{1}}\right)(\delta(x)) \\
& +(\delta(x))^{\left(\alpha \sigma_{2} / 2\right)-\lambda_{2}}\left(L_{2} b_{1}^{\sigma_{2}}\right)(\delta(x)) \\
\approx & (\delta(x))^{-\alpha / 2}\left(L_{1} b_{1}^{\sigma_{1}}\right)(\delta(x)) .
\end{aligned}
$$

Hence applying Lemma 11 with $\mu=\alpha / 2$, we obtain

$$
\begin{aligned}
G_{D}^{\alpha}[ & \left.a_{1} \theta^{\sigma_{1}}(\delta(\cdot))+a_{2} \theta^{\sigma_{2}}(\delta(\cdot))\right](x) \\
& \approx G_{D}^{\alpha}\left[(\delta(\cdot))^{-\alpha / 2}\left(L_{1} b_{1}^{\sigma_{1}}\right)(\delta(\cdot))\right](x) \\
& \approx(\delta(x))^{\alpha / 2} \int_{\delta(x)}^{\eta} \frac{\left(L_{1} b_{1}^{\sigma_{1}}\right)(s)}{s} d s \\
& \approx(\delta(x))^{\alpha / 2} b_{1}(\delta(x))=\theta(\delta(x)) .
\end{aligned}
$$

Case 5. $\lambda_{1}<(\alpha / 2)\left(1+\sigma_{1}\right)$.
We have $\theta(t)=t^{\alpha / 2}$. So

$$
a_{1}(x) \theta^{\sigma_{1}}(\delta(x)) \approx(\delta(x))^{-\left(\lambda_{1}-\left(\alpha \sigma_{1} / 2\right)\right)} L_{1}(\delta(x)) .
$$

Applying again Lemma 11 with $\mu=\lambda_{1}-\left(\alpha \sigma_{1} / 2\right)<(\alpha / 2)$, we obtain

$$
G_{D}^{\alpha}\left[a_{1} \theta^{\sigma_{1}}(\delta(\cdot))\right](x) \approx(\delta(x))^{\alpha / 2} .
$$

On the other hand, since $(\alpha / 2)<\left(\alpha-\lambda_{1}\right) /\left(1-\sigma_{1}\right) \leq(\alpha-$ $\left.\lambda_{2}\right) /\left(1-\sigma_{2}\right)$, then $\lambda_{1}<(\alpha / 2)\left(1+\sigma_{1}\right)$ and therefore

$$
G_{D}^{\alpha}\left[a_{2} \theta^{\sigma_{2}}(\delta(\cdot))\right](x) \approx(\delta(x))^{\alpha / 2} .
$$

Hence

$$
G_{D}^{\alpha}\left[a_{1} \theta^{\sigma_{1}}(\delta(\cdot))+a_{2} \theta^{\sigma_{2}}(\delta(\cdot))\right](x) \approx(\delta(x))^{\alpha / 2}=\theta(\delta(x)) .
$$

The proof is complete.

3.3. Proof of Theorem 4. Let $\sigma_{1}, \sigma_{2} \in(-1,1)$, assume $(\mathrm{H})$, and consider $v:=G_{\alpha}^{D}\left[a_{1} \theta^{\sigma_{1}}(\delta(\cdot))+a_{2} \theta^{\sigma_{2}}(\delta(\cdot))\right]$. Using Theorem 3, there exists $m>1$ such that

$$
\frac{1}{m} v(x) \leq \theta(\delta(x)) \leq m v(x) .
$$

Put $\sigma:=\max \left(\left|\sigma_{1}\right|,\left|\sigma_{2}\right|\right), c:=m^{\sigma /(1-\sigma)}$ and consider the set

$$
\begin{aligned}
& \Gamma:=\{\omega \in C_{0}(D): \frac{1}{c}(\delta(x))^{1-(\alpha / 2)} v(x) \leq \omega(x) \\
&\left.\leq c(\delta(x))^{1-(\alpha / 2)} v(x), x \in D\right\} .
\end{aligned}
$$

Let $h$ be the function given by (39). Since $a_{1}(x) \theta^{\sigma_{1}}(\delta(x))+$ $a_{2}(x) \theta^{\sigma_{2}}(\delta(x)) \approx(\delta(x))^{(\alpha / 2)-1} h(\delta(x))$ and the function $x \rightarrow$ $h(\delta(x))$ is in $K_{\alpha}(D)$, it follows by Proposition 9 that $x \rightarrow$ $(\delta(x))^{1-(\alpha / 2)} v(x)$ is in $C_{0}(D)$. So $\Gamma$ is a nonempty, closed, bounded, and convex set in $C_{0}(D)$. Define the operator $T$ on $\Gamma$ by

$$
\begin{aligned}
T \omega(x):=(\delta(x))^{1-(\alpha / 2)} G_{\alpha}^{D}( & \left((\delta(\cdot))^{(\alpha / 2)-1} \omega\right)^{\sigma_{1}} a_{1} \\
& \left.+\left((\delta(\cdot))^{(\alpha / 2)-1} \omega\right)^{\sigma_{2}} a_{2}\right)(x) .
\end{aligned}
$$

We will prove that $T$ has a fixed point. Since there exists a constant $c>0$ such that for all $\omega \in \Gamma$ we have

$$
\begin{aligned}
& \mid a_{1}(x)\left((\delta(x))^{(\alpha / 2)-1} \omega(x)\right)^{\sigma_{1}} \\
& \quad+a_{2}(x)\left((\delta(x))^{(\alpha / 2)-1} \omega(x)\right)^{\sigma_{2}} \mid \leq c(\delta(x))^{(\alpha / 2)-1} h(\delta(x)),
\end{aligned}
$$

where the function $x \rightarrow h(\delta(x))$ is in $K_{\alpha}(D)$, it follows that $T(\Gamma) \subset \Lambda_{h(\delta(\cdot))}$, where $\Lambda_{h(\delta(\cdot))}$ is given by (29). Therefore by Proposition 9, the family of functions $\{x \rightarrow T \omega(x), \omega \in \Gamma\}$ is relatively compact in $C_{0}(D)$.

Next, we will prove that $T$ maps $\Gamma$ into itself. 
Indeed, by using (60) we have for all $\omega \in \Gamma$

$$
\begin{aligned}
& G_{\alpha}^{D}\left(a_{1}\left((\delta(\cdot))^{(\alpha / 2)-1} \omega\right)^{\sigma_{1}}+a_{2}\left((\delta(\cdot))^{(\alpha / 2)-1} \omega\right)^{\sigma_{2}}\right) \\
& \quad \leq G_{\alpha}^{D}\left(a_{1} c^{\sigma} m^{\sigma} \theta^{\sigma_{1}}(\delta(\cdot))+a_{2} c^{\sigma} m^{\sigma} \theta^{\sigma_{2}}(\delta(\cdot))\right) \\
& \quad=c v .
\end{aligned}
$$

On the other hand, we have

$$
\begin{aligned}
& G_{\alpha}^{D}\left(a_{1}\left((\delta(\cdot))^{(\alpha / 2)-1} \omega\right)^{\sigma_{1}}+a_{2}\left((\delta(\cdot))^{(\alpha / 2)-1} \omega\right)^{\sigma_{2}}\right) \\
& \quad \geq G_{\alpha}^{D}\left(a_{1} c^{-\sigma} m^{-\sigma} \theta^{\sigma_{1}}(\delta(\cdot))+a_{2} c^{-\sigma} m^{-\sigma} \theta^{\sigma_{2}}(\delta(\cdot))\right) \\
& \quad=\frac{1}{c} v .
\end{aligned}
$$

This implies that $T(\Gamma) \subset \Gamma$.

Now, we will prove the continuity of the operator $T$ in $\Gamma$ in the supremum norm. Let $\left(\omega_{k}\right)_{k \in \mathbb{N}}$ be a sequence in $\Gamma$ which converges uniformly to a function $\omega$ in $\Gamma$. Then, for each $x \in$ $D$, we have

$$
\begin{aligned}
& \quad\left|T \omega_{k}(x)-T \omega(x)\right| \\
& \leq(\delta(x))^{1-(\alpha / 2)} G_{\alpha}^{D}\left(a_{1}(\delta(.))^{((\alpha / 2)-1) \sigma_{1}}\left|\omega_{k}^{\sigma_{1}}-\omega^{\sigma_{1}}\right|\right. \\
& \left.\quad+a_{2}(\delta(\cdot))^{((\alpha / 2)-1) \sigma_{2}}\left|\omega_{k}^{\sigma_{2}}-\omega^{\sigma_{2}}\right|\right)(x) .
\end{aligned}
$$

On the other hand, by similar arguments to the previous ones, we have

$$
\begin{aligned}
a_{1}(x) & (\delta(x))^{((\alpha / 2)-1) \sigma_{1}}\left|\omega_{k}^{\sigma_{1}}-\omega^{\sigma_{1}}\right|(x) \\
& +a_{2}(x)(\delta(x))^{((\alpha / 2)-1) \sigma_{2}}\left|\omega_{k}^{\sigma_{2}}-\omega^{\sigma_{2}}\right|(x) \\
\leq & c(\delta(x))^{(\alpha / 2)-1} h(\delta(x)) .
\end{aligned}
$$

We conclude by Proposition 9 and the dominated convergence theorem that, for all $x \in D$,

$$
T \omega_{k}(x) \longrightarrow T \omega(x) \text { as } k \longrightarrow+\infty .
$$

Consequently, as $T(\Gamma)$ is relatively compact in $C_{0}(D)$, we deduce that the pointwise convergence implies the uniform convergence; namely,

$$
\left\|T \omega_{k}-T \omega\right\|_{\infty} \longrightarrow 0 \text { as } k \longrightarrow+\infty .
$$

Therefore, $T$ is a compact operator from $\Gamma$ into itself. So the Schauder fixed-point theorem implies the existence of $\omega \in \Gamma$ such that

$$
\begin{aligned}
\omega(x)=(\delta(x))^{1-(\alpha / 2)} G_{\alpha}^{D}( & a_{1}\left((\delta(\cdot))^{(\alpha / 2)-1} \omega\right)^{\sigma_{1}} \\
& \left.+a_{2}\left((\delta(\cdot))^{(\alpha / 2)-1} \omega\right)^{\sigma_{2}}\right)(x) .
\end{aligned}
$$

Put $u(x)=(\delta(x))^{(\alpha / 2)-1} \omega(x)$. Then $u$ is continuous and satisfies

$$
u(x)=G_{\alpha}^{D}\left(a_{1} u^{\sigma_{1}}+a_{2} u^{\sigma_{2}}\right)(x) .
$$

Hence by Lemma 12 and Theorem 3, $u$ is a required solution.
Next, we aim at proving the uniqueness in the cone

$$
S:=\{u \in C(D): u(x) \approx \theta(\delta(x))\} .
$$

Let $u$ and $v$ be two solutions of (14) in S. Then there exists a constant $m>1$ such that

$$
\frac{1}{m} \leq \frac{u}{v} \leq m
$$

This implies that the set

$$
J=\left\{t \in(1, \infty), \frac{1}{t} v \leq u \leq t v\right\}
$$

is not empty. Let $c_{0}:=\inf J$ and put $w=v-c_{0}^{-\sigma} u$ with $\sigma=$ $\max \left(\left|\sigma_{1}\right|,\left|\sigma_{2}\right|\right)$.

We claim that $c_{0}=1$. Indeed, assume that $c_{0}>1$; then by using Lemma 12, we deduce that

$$
\begin{aligned}
w & =G_{\alpha}^{D}\left(a_{1}\left(v^{\sigma_{1}}-c_{0}^{-\sigma} u^{\sigma_{1}}\right)+a_{2}\left(v^{\sigma_{2}}-c_{0}^{-\sigma} u^{\sigma_{2}}\right)\right) \\
& \geq 0 \quad \text { in } D,
\end{aligned}
$$

which implies that

$$
v \geq c_{0}^{-\sigma} u
$$

By symmetry, we deduce that

$$
v \leq c_{0}^{\sigma} u
$$

So $c_{0}^{\sigma} \in J$. Since $\sigma<1$, then we have $c_{0}^{\sigma}<c_{0}$. This is a contradiction to the fact that $c_{0}:=\inf J$. Hence $c_{0}=1$ and so $u=v$. This completes the proof.

Example 14. Let $\sigma_{1} \in(-1,0)$, let $\sigma_{2} \in(0,1)$, and put $d=$ $\operatorname{diam}(D)$. For $i \in\{1,2\}$, let $a_{i} \in C_{\mathrm{loc}}^{\gamma}(D), 0<\gamma<1$, satisfying

$$
\begin{gathered}
a_{1}(x) \approx(\delta(x))^{-\lambda_{1}}\left(\log \left(\frac{3 d}{\delta(x)}\right)\right)^{-1}, \\
a_{2}(x) \approx(\delta(x))^{-\lambda_{2}},
\end{gathered}
$$

where $\lambda_{i}<(\alpha / 2)\left(1+\sigma_{i}\right)+1-\sigma_{i}$, such that $\left(\alpha-\lambda_{1}\right) /\left(1-\sigma_{1}\right) \leq$ $\left(\alpha-\lambda_{2}\right) /\left(1-\sigma_{2}\right)$. Then using Theorem 4 , problem (14) has a unique positive continuous solution $u$ in $D$ satisfying the following estimates:

(i) if $\left(\alpha-\lambda_{1}\right) /\left(1-\sigma_{1}\right)<\left(\alpha-\lambda_{2}\right) /\left(1-\sigma_{2}\right)$ and $(\alpha / 2)(1+$ $\left.\sigma_{1}\right)<\lambda_{1}$, then, for $x \in D$,

$$
u(x) \approx(\delta(x))^{\left(\alpha-\lambda_{1}\right) /\left(1-\sigma_{1}\right)}\left(\log \left(\frac{3 d}{\delta(x)}\right)\right)^{-1 /\left(1-\sigma_{1}\right)} ;
$$

(ii) if $\left(\alpha-\lambda_{1}\right) /\left(1-\sigma_{1}\right)=\left(\alpha-\lambda_{2}\right) /\left(1-\sigma_{2}\right)$ and $(\alpha / 2)(1+$ $\left.\sigma_{1}\right)<\lambda_{1}$, then, for $x \in D$,

$$
u(x) \approx(\delta(x))^{\left(\alpha-\lambda_{1}\right) /\left(1-\sigma_{1}\right)} ;
$$

(iii) if $\lambda_{1}=(\alpha / 2)\left(1+\sigma_{1}\right)$ and $\lambda_{2}=(\alpha / 2)\left(1+\sigma_{2}\right)$, then, for $x \in D$,

$$
u(x) \approx(\delta(x))^{\alpha / 2}\left(\log \left(\frac{3 d}{\delta(x)}\right)\right)^{1 /\left(1-\sigma_{2}\right)} ;
$$


(iv) if $\lambda_{1}=(\alpha / 2)\left(1+\sigma_{1}\right)$ and $\lambda_{2}<(\alpha / 2)\left(1+\sigma_{2}\right)$, then, for $x \in D$,

$$
u(x) \approx(\delta(x))^{\alpha / 2}\left(\log \circ \log \left(\frac{3 d}{\delta(x)}\right)\right)^{1 /\left(1-\sigma_{1}\right)} ;
$$

(v) if $\lambda_{1}<(\alpha / 2)\left(1+\sigma_{1}\right)$, then, for $x \in D$,

$$
u(x) \approx(\delta(x))^{(\alpha / 2)} .
$$

\section{Acknowledgments}

The authors are thankful to the referees for their careful reading of the paper and for their helpful comments and suggestions. The research of Imed Bachar is supported by NPST Program of King Saud University, Project no. 11MAT1716-02.

\section{References}

[1] N. S. Landkof, Foundations of Modern Potential Theory, Springer, New York, NY, USA, 1972.

[2] E. M. Stein, Singular integrals and differentiability properties of functions, vol. 30 of Princeton Mathematical Series, Princeton University Press, Princeton, NJ, USA, 1970.

[3] D. Applebaum, Lévy Processes and Stochastic Calculus, vol. 116 of Cambridge Studies in Advanced Mathematics, Cambridge University Press, Cambridge, UK, 2nd edition, 2009.

[4] J. Bertoin, Lévy Processes, vol. 121 of Cambridge Tracts in Mathematics, Cambridge University Press, Cambridge, UK, 1996.

[5] E. Valdinoci, "From the long jump random walk to the fractional Laplacian," Boletín de la Sociedad Española de Matemática Aplicada, no. 49, pp. 33-44, 2009.

[6] L. Abdelouhab, J. L. Bona, M. Felland, and J.-C. Saut, "Nonlocal models for nonlinear, dispersive waves," Physica D, vol. 40, no. 3, pp. 360-392, 1989.

[7] K. Bogdan and T. Byczkowski, "Potential theory for the $\alpha$-stable Schrödinger operator on bounded Lipschitz domains," Studia Mathematica, vol. 133, no. 1, pp. 53-92, 1999.

[8] K. Bogdan, "Representation of $\alpha$-harmonic functions in Lipschitz domains," Hiroshima Mathematical Journal, vol. 29, no. 2, pp. 227-243, 1999.

[9] L. Caffarelli and L. Silvestre, "An extension problem related to the fractional Laplacian," Communications in Partial Differential Equations, vol. 32, no. 7-9, pp. 1245-1260, 2007.

[10] A. Elgart and B. Schlein, "Mean field dynamics of boson stars," Communications on Pure and Applied Mathematics, vol. 60, no. 4, pp. 500-545, 2007.

[11] J. Fröhlich and E. Lenzmann, "Blowup for nonlinear wave equations describing boson stars," Communications on Pure and Applied Mathematics, vol. 60, no. 11, pp. 1691-1705, 2007.

[12] L. A. Caffarelli, "Further regularity for the Signorini problem," Communications in Partial Differential Equations, vol. 4, no. 9, pp. 1067-1075, 1979.

[13] A. Signorini, "Questioni di elasticità non linearizzata e semilinearizzata," vol. 18, pp. 95-139, 1959.

[14] L. A. Caffarelli and A. Vasseur, "Drift diffusion equations with fractional diffusion and the quasi-geostrophic equation," Annals of Mathematics, vol. 171, no. 3, pp. 1903-1930, 2010.
[15] I. Athanasopoulos, L. A. Caffarelli, and S. Salsa, "The structure of the free boundary for lower dimensional obstacle problems," American Journal of Mathematics, vol. 130, no. 2, pp. 485-498, 2008.

[16] L. A. Caffarelli, S. Salsa, and L. Silvestre, "Regularity estimates for the solution and the free boundary of the obstacle problem for the fractional Laplacian," Inventiones Mathematicae, vol. 171, no. 2, pp. 425-461, 2008.

[17] M. Kassmann, "A priori estimates for integro-differential operators with measurable kernels," Calculus of Variations and Partial Differential Equations, vol. 34, no. 1, pp. 1-21, 2009.

[18] L. Silvestre, "Regularity of the obstacle problem for a fractional power of the Laplace operator," Communications on Pure and Applied Mathematics, vol. 60, no. 1, pp. 67-112, 2007.

[19] S. Abe and S. Thurner, "Anomalous diffusion in view of Einstein's 1905 theory of Brownian motion," Physica A, vol. 356, no. 2-4, pp. 403-407, 2005.

[20] M. Jara, "Nonequilibrium scaling limit for a tagged particle in the simple exclusion process with long jumps," Communications on Pure and Applied Mathematics, vol. 62, no. 2, pp. 198-214, 2009.

[21] A. Mellet, S. Mischler, and C. Mouhot, "Fractional diffusion limit for collisional kinetic equations," Archive for Rational Mechanics and Analysis, vol. 199, no. 2, pp. 493-525, 2011.

[22] L. Vlahos, H. Isliker, Y. Kominis, and K. Hizonidis, "Normal and anomalous diffusion: a tutorial," in Order and Chaos, T. Bountis, Ed., vol. 10, Patras University Press, Patras, Greece, 2008.

[23] H. Weitzner and G. M. Zaslavsky, "Some applications of fractional equations. Chaotic transport and complexity in classical and quantum dynamics," Communications in Nonlinear Science and Numerical Simulation, vol. 8, no. 3-4, pp. 273-281, 2003.

[24] J. L. Vazquez, "Nonlinear diffusion with fractional Laplacian operators," Non-Linear Partial Differential Equations, vol. 7, pp. 271-298, 2012.

[25] E. Di Nezza, G. Palatucci, and E. Valdinoci, "Hitchhiker's guide to the fractional Sobolev spaces," Bulletin des Sciences Mathématiques, vol. 136, no. 5, pp. 521-573, 2012.

[26] R. Chemmam, H. Mâagli, and S. Masmoudi, "On a new Kato class and positive solutions of Dirichlet problems for the fractional Laplacian in bounded domains," Nonlinear Analysis. Theory, Methods \& Applications, vol. 74, no. 5, pp. 1555-1576, 2011.

[27] H. Mâagli and M. Zribi, "On a new Kato class and singular solutions of a nonlinear elliptic equation in bounded domains of $\mathbb{R}^{n}$," Positivity, vol. 9, no. 4, pp. 667-686, 2005.

[28] R. Chemmam, H. Mâagli, and S. Masmoudi, "Boundary behavior of positive solutions of a semilinear fractional Dirichlet problem," Journal of Abstract Differential Equations and Applications, vol. 3, no. 2, pp. 75-90, 2012.

[29] F. C. Cîrstea and V. Rădulescu, "Nonlinear problems with boundary blow-up: a Karamata regular variation theory approach," Asymptotic Analysis, vol. 46, no. 3-4, pp. 275-298, 2006.

[30] V. Marić, Regular Variation and Differential Equations, vol. 1726 of Lecture Notes in Mathematics, Springer, Berlin, Germany, 2000.

[31] E. Seneta, Regularly Varying Functions, vol. 508, Springer, Berlin, Germany, 1976.

[32] S. Gontara, H. Mâagli, S. Masmoudi, and S. Turki, "Asymptotic behavior of positive solutions of a singular nonlinear Dirichlet problem," Journal of Mathematical Analysis and Applications, vol. 369, no. 2, pp. 719-729, 2010. 
[33] H. Mâagli, "Asymptotic behavior of positive solutions of a semilinear Dirichlet problem," Nonlinear Analysis. Theory, Methods \& Applications, vol. 74, no. 9, pp. 2941-2947, 2011.

[34] A. Ambrosetti, H. Brezis, and G. Cerami, "Combined effects of concave and convex nonlinearities in some elliptic problems," Journal of Functional Analysis, vol. 122, no. 2, pp. 519-543, 1994.

[35] L. Boccardo, "A Dirichlet problem with singular and supercritical nonlinearities," Nonlinear Analysis. Theory, Methods \& Applications, vol. 75, no. 12, pp. 4436-4440, 2012.

[36] R. Chemmam, H. Mâagli, S. Masmoudi, and M. Zribi, "Combined effects in nonlinear singular elliptic problems in a bounded domain," Advances in Nonlinear Analysis, vol. 1, no. 4, pp. 301-318, 2012.

[37] V. Rădulescu and D. Repovš, "Combined effects in nonlinear problems arising in the study of anisotropic continuous media," Nonlinear Analysis. Theory, Methods \& Applications, vol. 75, no. 3, pp. 1524-1530, 2012.

[38] S. Yijing and L. Shujie, "Some remarks on a superlinear-singular problem: estimates of $\lambda^{*}$," Nonlinear Analysis. Theory, Methods \& Applications, vol. 69, no. 8, pp. 2636-2650, 2008.

[39] Y. Sun, S. Wu, and Y. Long, "Combined effects of singular and superlinear nonlinearities in some singular boundary value problems," Journal of Differential Equations, vol. 176, no. 2, pp. 511-531, 2001.

[40] Z.-Q. Chen and R. Song, "Estimates on Green functions and Poisson kernels for symmetric stable processes," Mathematische Annalen, vol. 312, no. 3, pp. 465-501, 1998.

[41] T. Kulczycki, "Properties of Green function of symmetric stable processes," Probability and Mathematical Statistics, vol. 17, pp. 339-364, 1997. 


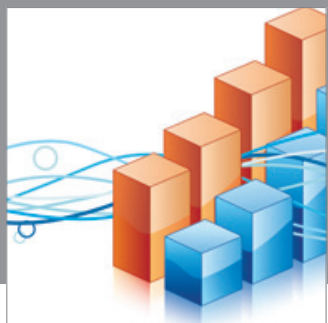

Advances in

Operations Research

mansans

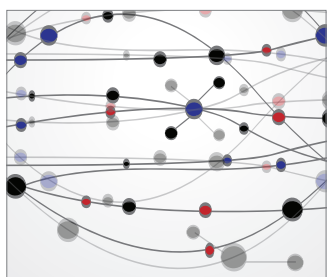

The Scientific World Journal
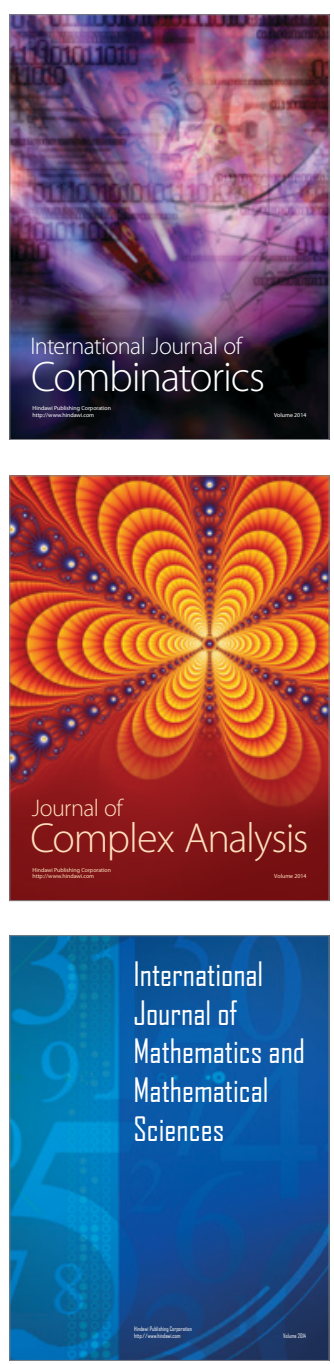
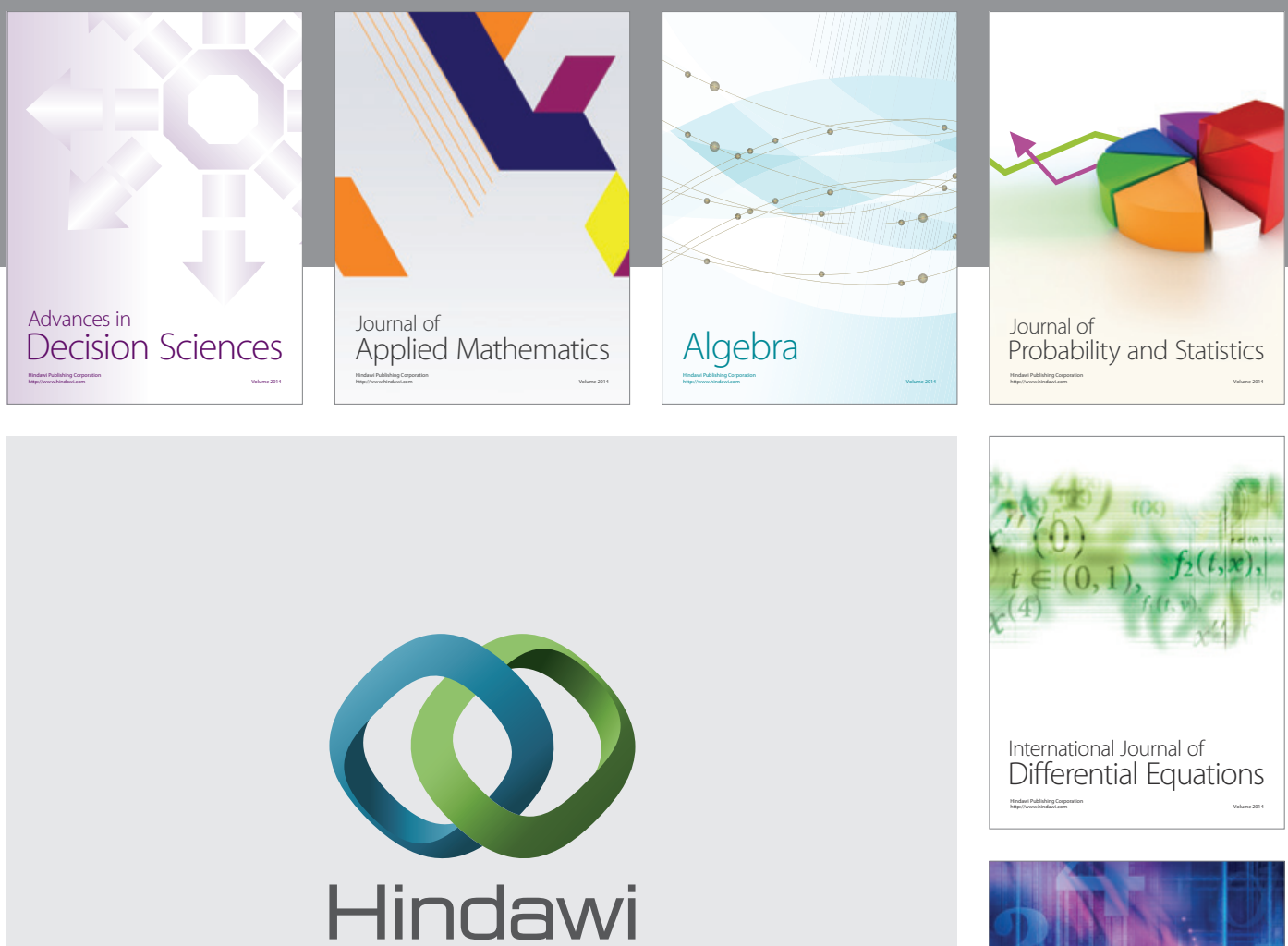

Submit your manuscripts at http://www.hindawi.com
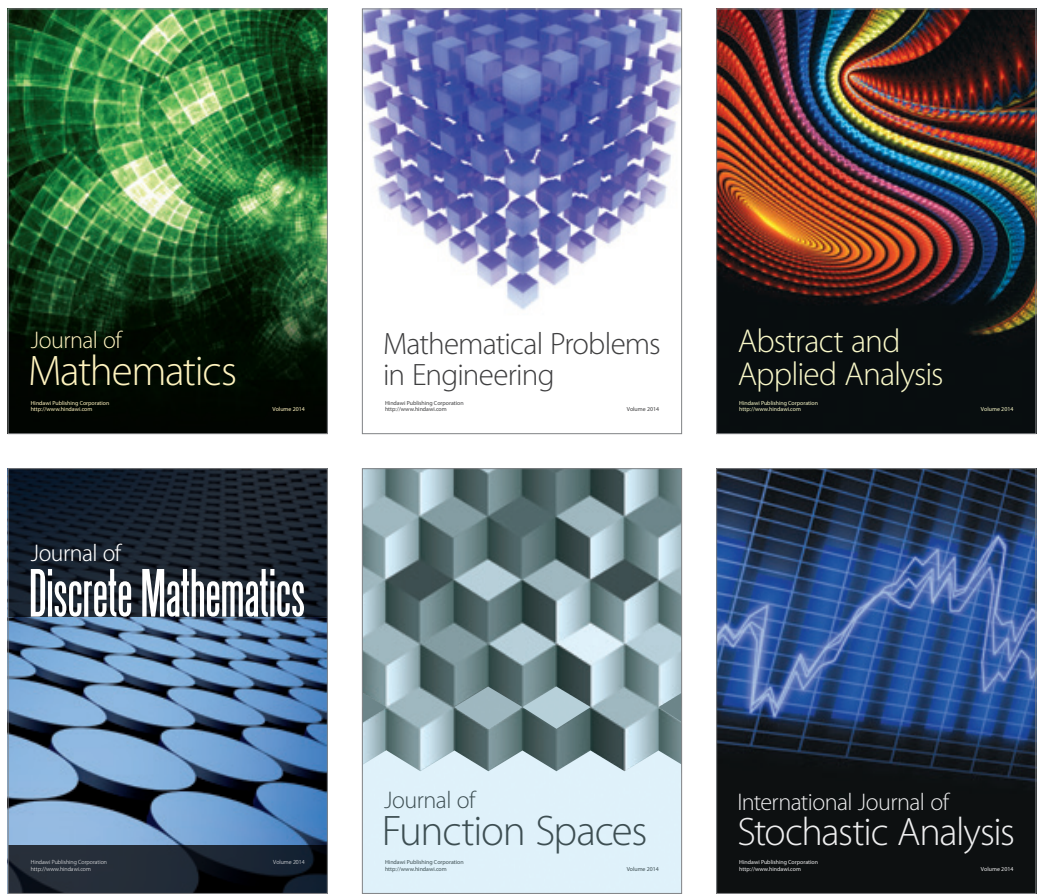

Journal of

Function Spaces

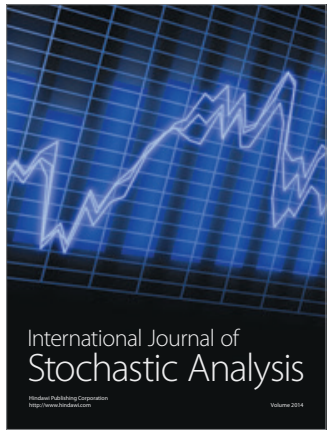

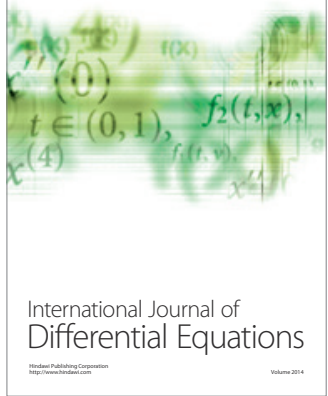
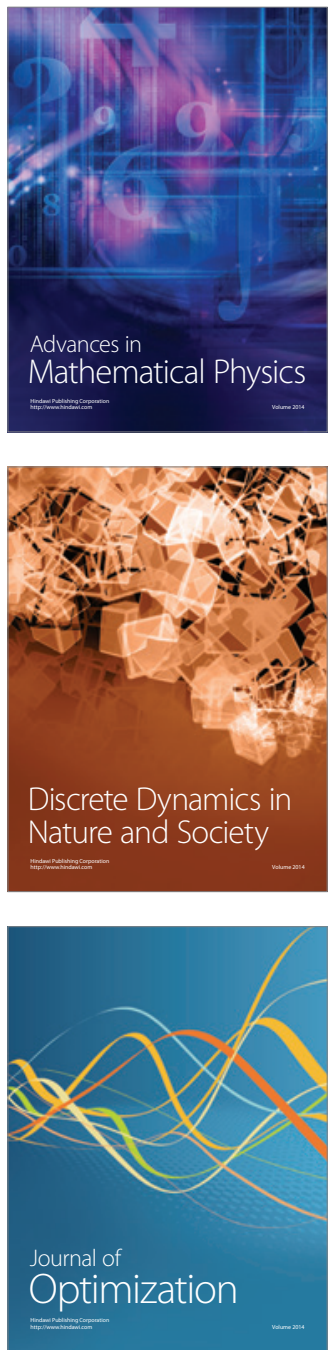